FACTA UNIVERSITATIS

Series: Mechanical Engineering Vol. 14, N ${ }^{\mathrm{o}}$ 1, 2016, pp. 75 - 88

Original scientific paper

\title{
BENDING VIBRATION AND STABILITY \\ OF A MULTIPLE-NANOBEAM SYSTEM INFLUENCED BY TEMPERATURE CHANGE
}

\author{
UDC 534.1
}

\section{Danilo Karličić ${ }^{1}$, Sanja Ožvat ${ }^{2}$, Milan Cajić ${ }^{3}$, Predrag Kozić ${ }^{1}$, Ratko Pavlović ${ }^{1}$}

\author{
${ }^{1}$ Faculty of Mechanical Engineering, University of Niš \\ ${ }^{2}$ Faculty of Technical Sciences, University of Novi Sad \\ ${ }^{3}$ Mathematical Institute of the Serbian Academy of Science and Arts
}

\begin{abstract}
In this study, we analyzed the bending vibration and stability of a multiplenanobeam system (MNBS) coupled in elastic medium and influenced by temperature change and compressive axial load. The MNBS is modeled as the system consisting of a set of m identical and simply supported nanobeams mutually connected by Winkler's type elastic layers. According to the Euler - Bernoulli beam and nonlocal thermo-elasticity theory, the system of $m$ coupled partial differential equations is derived and solved by means of the method of separation of variables as well as the trigonometric one. Analytical solutions for natural frequencies and critical buckling loads of elastic MNBS are obtained. The effects of nonlocal parameter, temperature change and the number of nanobeams on the natural frequencies and the buckling loads are investigated through numerical examples. Thus, this work can represent a starting point to examine dynamical behavior and design of complex nanobeam structures, nanocomposites and nanodevices under the influence of various physical fields.
\end{abstract}

Key Words: Nonlocal Elasticity, Vibration, Stability, Multiple-nanobeam System

\section{INTRODUCTION}

The nonlocal continuum theory has recently been widely used for the study of the mechanical behavior of nanobeams such as bending, vibration and buckling. Such theoretical observations of nanostructures may be important for nanoengineering practice

Received September 30, 2015 / Accepted January 12, 2015

Corresponding author: Danilo Karličić

Faculty of Mechanical Engineering, University of Niš, A. Medvedeva 14, 18000 Niš, Serbia,

E-mail: danilo.karlicic@ masfak.ni.ac.rs 
in the development of new nanodevices. Other methods of nanostructure examination, such as experimental [1-3] or atomistic simulations [4-7] can be expensive, taking into consideration time or computational resources needed to gather important data about the mechanical behavior of nanostructures. Thus, the modified continuum theories are increasingly gaining in importance for the mathematical modeling of dynamical behavior of systems and structures on small-scales [8-10]. In this study, the nanobeam may represent various types of tube-like nanomaterials such as carbon nanotubes (CNT) [11], zinc-oxide nanotubes $(\mathrm{ZnO})$ [12], boron-nitride $(\mathrm{BN})$ nanotubes [13, 14], etc.

According to the experimental analysis and atomistic theories, it has been shown that size-effects play an important role in describing the physical and mechanical properties of structures and systems on the nano-scale level. Due to the existence of such effects, the classical continuum theories need to be reformulated to take into account these smallscale effects. Aforementioned can be done by introducing the nonlocality in the space domain, modifying the corresponding constitutive equation and introducing the material parameter which takes into account the effects of length scale and influence of interatomic forces. One of the first scale-dependent continuum theories, which takes into account nonlocal effects, is proposed by Eringen and co-workers [15-17] also known as nonlocal elasticity theory. According to Eringen [18], the nonlocal theory gives great approximation for a large class of problems in nano-systems, where the influence of length-scales is very noticeable. The nonlocal elasticity theory in nanomechanics was first considered in the paper by Peddieson et al. [19] on the static behavior study of nanobeams. The authors have investigated the nonlocal Euler-Bernoulli beam deflection equation for different boundary conditions and applications on the micro actuators. Reddy and Pang [20] reformulated the equations of motion for Euler-Bernoulli and Timoshenko beam theories, by using the nonlocal theory, and applied them to evaluate static bending, vibrations, and buckling response of the nanobeams with various boundary conditions. Recently, nonlocal models for the Euler-Bernoulli, Timoshenko, Reddy and Levinson beam theories are formulated by Reddy [21] based on the Hamilton's principle and nonlocal constitutive relation of Eringen.

Thermal effects studies are important to round up the knowledge of the mechanical behavior of structures, regardless of scale. Zhang et al. [22, 23] derived equations of motion and stability equation for double-walled and multi-walled carbon nanotubes, respectively, and solved them by using the separation of variables method. The authors obtained analytical solutions for natural frequencies and critical axial buckling strain, and examined the effects of thermal and other physical and geometrical parameters on the dynamic and stability behavior of the proposed system. In the work by Janghorban [24], the static analysis of microbeams was conducted, based on the nonlocal thermal elasticity theory and using the differential quadrature (DQ) and harmonic differential quadrature (HDQ) methods. The author has presented a variety of numerical results to show the effects of temperature change on bending of microbeams, by choosing different combinations of aspect ratios and boundary conditions. Murmu and Pradhan $[25,26]$ have used a nonlocal constitutive relation in modeling of carbon nanotubes embedded in an elastic medium with thermal effects included. They obtained approximated solutions for the natural frequencies and the critical buckling force by using the differential quadrature method for simply supported carbon nanotubes. In addition, they performed numerical experiments for two types of the thermal expansion parameters corresponding to the 
lower and higher environmental temperature. It has been concluded that in the case of low or room temperature, the nonlocal critical buckling load and the natural frequencies increase as temperature rises. In the case of higher temperatures, the nonlocal critical buckling load and the natural frequencies decrease for an increase of temperature.

In this paper, we used the nonlocal thermo-elastic theory to investigate the free banding vibration and stability behavior of MNBS embedded in Winkler's type of elastic medium. It is assumed that all nanobeams in MNBS are simply supported, and are with the same material and geometric properties. The set of $\mathrm{m}$ equations of motion is obtained by using the Newton second low and nonlocal thermo-elastic constitutive relation based on the Euler-Bernoulli beam theory. The closed form solutions for the natural frequencies and the critical buckling loads are derived by using the separation of variables method and the trigonometric method for different number of nanobeams in MNBS. Special attention is given to the effects of temperature changes and nonlocal parameter on the vibration and stability behavior of MNBS.

By searching the literature, one can find that the dynamic behavior of systems, consisting of an arbitrary number of nanobeams, embedded in a certain type of medium, considering temperature effects, has not been investigated yet. This work can be used as an analytical benchmark study in the future research on vibration and stability behavior of other types of complex multi-nanostructure systems such as coupled photonic crystal nanobeam cavities [32] and nanocomposites [33].

\section{PROBLEM FORMULATION}

\subsection{Constitutive relation}

In this section, we will consider the basic equations of nonlocal elasticity and viscoelasticity in the general and two-dimensional case. Eringen [15] derived a constitutive relation for nonlocal stress tensor at a point $x$ in an integral form, based on the assumption that the stress at a point is a function of strains at all other points of an elastic body. The fundamental form of the nonlocal elastic constitutive relation for a three-dimensional linear, homogeneous, isotropic body is expressed as:

$$
\begin{gathered}
\boldsymbol{\sigma}_{i j}(x)=\int \boldsymbol{\alpha}\left(\left|x-x^{\prime}\right|, \tau\right) C_{i j k l} \boldsymbol{\varepsilon}_{k l}\left(x^{\prime}\right) d V\left(x^{\prime}\right), \\
\boldsymbol{\sigma}_{i j, j}(x)=0 \\
\boldsymbol{\varepsilon}_{i j}(x)=\frac{1}{2}\left(u_{i, j}+u_{j, i j}\right)
\end{gathered}
$$

where $C_{i j k l}$ is the elastic modulus tensor for classical isotropic elasticity; $\sigma_{i j}$ and $\boldsymbol{\varepsilon}_{i j}$ are stress and strain tensors, respectively, and $u_{i}$ is the displacement vector. With $\boldsymbol{\alpha}\left(\left|x-x^{\prime}\right|, \tau\right)$ we denote the nonlocal modulus or attenuation function, which incorporates nonlocal effects into the constitutive equation at a reference point $x$, produced by the local strain at a source $x^{\prime}$. The above absolute value of difference $\left|x-x^{\prime}\right|$ denotes the Euclidean metric. Parameter $\tau$ is $\tau=\left(e_{0} a\right) / l$, where $l$ is the external characteristic length (crack length, wave length), $a$ describes the internal characteristic length (lattice parameter, 
granular size and distance between $\mathrm{C}-\mathrm{C}$ bounds) and $e_{0}$ is the constant appropriate to each material that can be identified from atomistic simulations or by using the dispersive curve of the Born-Karman model of lattice dynamics. However, some difficulties may arise in searching the analytical solutions for structural mechanics problems when the constitutive relation is in integral form. Thus, for the sake of simplicity, Eringen [18] proposed the constitutive relation in a differential form, which, for one-dimensional problem, is of the form:

$$
\boldsymbol{\sigma}_{x x}-\boldsymbol{\mu} \frac{\partial^{2} \boldsymbol{\sigma}_{x x}}{\partial x^{2}}=E \boldsymbol{\varepsilon}_{x x},
$$

where $E$ and $G$ are the elastic and shear modulus of the beam; $\mu=\left(e_{0} a\right)^{2}$ is the nonlocal parameter, $\boldsymbol{\sigma}_{x x}$ is the normal nonlocal stress and $\boldsymbol{\varepsilon}_{x x}=-z \partial w / \partial x$ is the axial strain. In the present work, free vibration and the critical buckling load analysis of MNBS is carried out by assuming $e_{0} a$ to be in the range $0-2[\mathrm{~nm}]$. The nonlocal thermo-elastic constitutive relation is a combination of nonlocal elasticity and thermo-elasticity theory, as given in the paper [25]. For one-dimensional case, the nonlocal thermo-elastic constitutive relation is of the following form:

$$
\boldsymbol{\sigma}_{x x}-\boldsymbol{\mu} \frac{\partial^{2} \boldsymbol{\sigma}_{x x}}{\partial x^{2}}=E \boldsymbol{\varepsilon}_{x x}-\frac{E \boldsymbol{\alpha}_{x} \boldsymbol{\theta}}{1-2 \boldsymbol{v}}
$$

where $\boldsymbol{\alpha}_{x}$ is the coefficient of thermal expansion in the direction of $x$ axis, $\boldsymbol{\theta}$ is the change of temperature and $v$ is the Poisson's ratio. For $\theta=0$ there is no influence of temperature and we return to the constitutive relation of nonlocal elasticity.

\subsection{Equations of motion}

Now we consider the system of $m$ axially loaded nanobeams, coupled through an elastic medium, as shown in Fig. 1. Further, we assume that MNBS is made of $m$ identical nanobeams of the same length $L$, elastic modulus $E$ and mass density $\rho$, uniform crosssection of area $A$ and moment of inertia $I$. Each nanobeam is under the influence of the same compressive axial load $F$. In addition, for the $i$-th nanobeam we assume only the transverse deflection denoted as $w_{i}(x, t)$ where $i=1,2$.. $m$. Further, we limited our analysis only to the case where the nonlocal Euler-Bernoulli beam theory is used for simply supported boundary conditions. According to the way of coupling of the first and the last nanobeam in MNBS, we can distinguish between two different types of MNBS, "Clamped-Chain and "Free-Chain" system [29, 30]. In this work, we used only "Clamped-Chain" MNBS, in which the first and the last nanobeam are coupled with fixed base through Winkler's type elastic layers of stiffness $k_{0}$ and $k_{m}$, respectively, as shown in Fig. 1b. The coupling conditions are $w_{0}=w_{m+1}=0$. Other nanobeams in MNBS are coupled through elastic layers of stiffness $k_{0}=k_{1}=\ldots=k_{i}=\ldots=k_{m-1}=k_{m}=k$ representing the Winkler's type of elastic medium (see Fig. 1b).

According to the D' Alembert's principle, from the equilibrium equation for differential element of the $i$-th nanobeam we can write the following differential equation:

$$
\frac{\partial^{2} M_{f}}{\partial x^{2}}+(N-F) \frac{\partial^{2} w_{i}}{\partial x^{2}}+q_{i}+q_{i-1}=\rho A \frac{\partial^{2} w_{i}}{\partial t^{2}}, \quad i=1,2, \ldots, m,
$$


where $M_{f}$ and $N$ are stress resultants defined as:

$$
M_{f}=\int_{0}^{A} z \boldsymbol{\sigma}_{x x} d A, \quad N=\int_{0}^{A} \boldsymbol{\sigma}_{x x} d A=-\frac{E A \boldsymbol{\alpha}_{x} \boldsymbol{\theta}}{1-2 \mathbf{v}} .
$$

and the influence of the Winkler's type elastic medium expressed as external load is:

$$
q_{i}=k_{i}\left(w_{i+1}-w_{i}\right), \quad q_{i-1}=k_{i-1}\left(w_{i}-w_{i-1}\right) .
$$

Governing equations of motions can be expressed in terms of displacement $w_{i}(x, t)$ for nonlocal thermo-elastic constitutive relation Eq. (3).

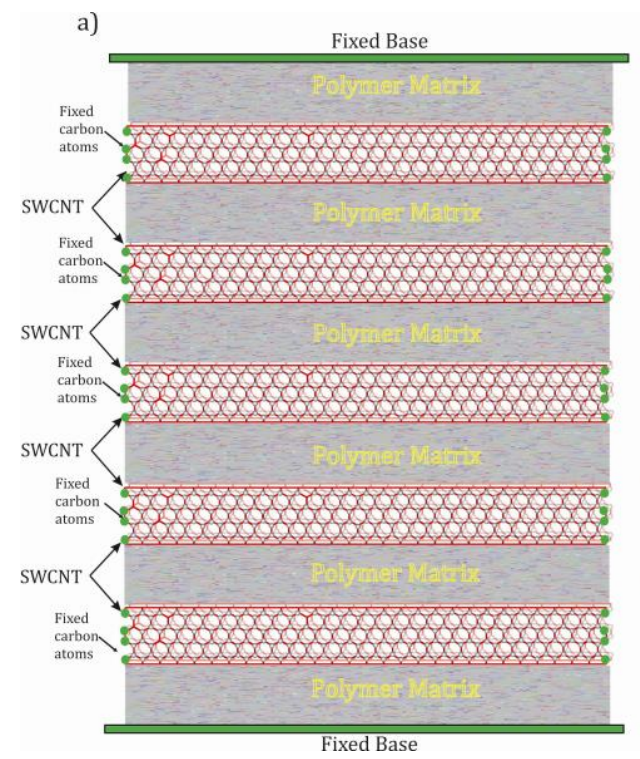

b)

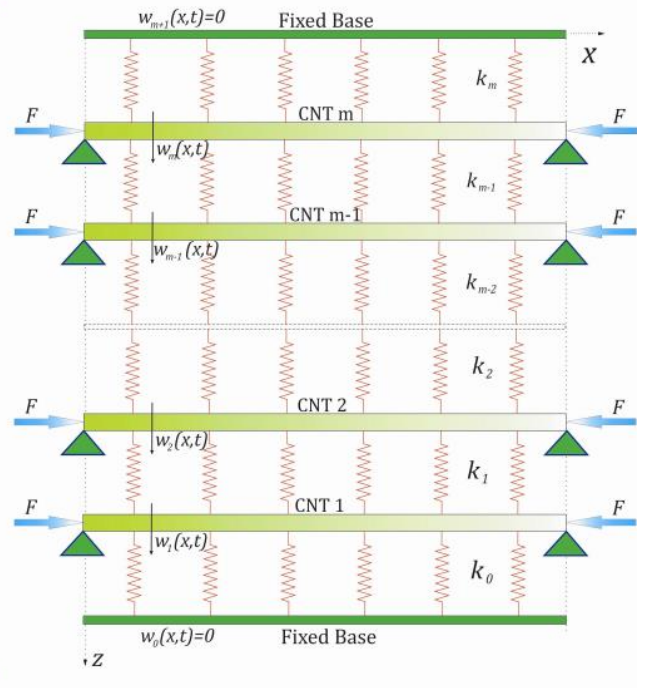

Fig. 1 The multiple-nanobeam system embedded in the Winkler's type of elastic medium: a) The physical model of the system of multiple SWCNTs embedded in a polymer matrix with atoms of SWCNTs fixed in such a manner that can be represented by simply supported boundary conditions [31], b) Mechanical model of multiplenanobeam system coupled in the "Clamped-Chain" system

Introducing Eqs. (4-6) into Eq. (3) we obtain the following system of equations of motion:

$$
\begin{gathered}
\boldsymbol{\rho} A \frac{\partial^{2} w_{i}}{\partial t^{2}}+(F-N) \frac{\partial^{2} w_{i}}{\partial x^{2}}+k_{i}\left(w_{i}-w_{i+1}\right)+k_{i-1}\left(w_{i}-w_{i-1}\right)+E I \frac{\partial^{4} w_{i}}{\partial x^{4}} \\
=\boldsymbol{\mu} \frac{\partial^{2}}{\partial x^{2}}\left[\boldsymbol{\rho} A \frac{\partial^{2} w_{i}}{\partial t^{2}}+(F-N) \frac{\partial^{2} w_{i}}{\partial x^{2}}+k_{i}\left(w_{i}-w_{i+1}\right)+k_{i-1}\left(w_{i}-w_{i-1}\right)\right], \quad i=1,2, . . m,
\end{gathered}
$$

or in the dimensionless form: 


$$
\begin{gathered}
\frac{\partial^{2} \bar{w}_{i}}{\partial \boldsymbol{\tau}^{2}}+\left(\bar{F}-\bar{N}_{\theta}\right) \frac{\partial^{2} \bar{w}_{i}}{\partial \zeta^{2}}+K_{i}\left(\bar{w}_{i}-\bar{w}_{i+1}\right)+K_{i-1}\left(\bar{w}_{i}-\bar{w}_{i-1}\right)+\frac{\partial^{4} \bar{w}_{i}}{\partial \zeta^{4}} \\
=\boldsymbol{\eta}^{2} \frac{\partial^{2}}{\partial \zeta^{2}}\left[\frac{\partial^{2} \bar{w}_{i}}{\partial \boldsymbol{\tau}^{2}}+\left(\bar{F}-\bar{N}_{\theta}\right) \frac{\partial^{2} \bar{w}_{i}}{\partial \zeta^{2}}+K_{i}\left(\bar{w}_{i}-\bar{w}_{i+1}\right)+K_{i-1}\left(\bar{w}_{i}-\bar{w}_{i-1}\right)\right], \quad i=1,2, . . m,
\end{gathered}
$$

where the dimensionless parameters are defined as:

$$
\begin{gathered}
K_{i}=k_{i} \frac{L^{4}}{E I}, \eta^{2}=\frac{\mu}{L^{2}}, \bar{N}_{\theta}=N \frac{L^{2}}{E I}, \bar{w}_{i}=\frac{w_{i}}{L}, \\
\zeta=\frac{x}{L}, \tau=\frac{t}{L^{2}} \sqrt{\frac{E I}{\rho A}}, \bar{F}=F \frac{L^{2}}{E I},
\end{gathered}
$$

The initial conditions in the general form and the boundary conditions in the dimensionless form for a simply supported $i$-th nonlocal Euler-Bernoulli nanobeam are given as:

$$
\begin{gathered}
\bar{w}_{i}(\zeta, 0)=\bar{w}_{i 0}(\zeta), \quad \frac{\partial \bar{w}_{i}(\zeta, 0)}{\partial \boldsymbol{\tau}}=\bar{v}_{i 0}(\zeta), \\
\bar{w}_{i}(0, \boldsymbol{\tau})=\bar{w}_{i}(1, \boldsymbol{\tau})=0, \quad \bar{M}_{f i}(0, \boldsymbol{\tau})=\bar{M}_{f i}(1, \boldsymbol{\tau})=0, \quad i=1,2, \ldots, m .
\end{gathered}
$$

\section{SOLUTION OF THE EQUATION OF MOTION}

Assumed solution of the system of partial differential equations Eq. (8) is in the form of trigonometric series, and this solution must satisfy the given boundary conditions Eq. $(10$ b), as:

$$
\bar{w}_{i}(\zeta, \boldsymbol{\tau})=\sum_{n=1}^{\infty} W_{i n} \sin \left(\boldsymbol{\alpha}_{n} \zeta\right) e^{j \Omega_{n} \tau}, \quad i=1,2, \ldots m
$$

where $j=\sqrt{-1}, \boldsymbol{\alpha}_{n}=n \pi(n=1,2, \ldots), W_{i n}(i=1,2, \ldots m)$ and $\Omega_{n}$ are the amplitudes and natural frequencies, respectively, in the dimensionless form. We substitute the assumed solution from Eq. (11) into the system of equations (8) and take into account the coupling conditions for "Clamped-Chain" systems in the dimensionless form $\bar{w}_{0}(\zeta, \tau)=\bar{w}_{m+1}(\zeta, \tau)=0$. Further, assume that all stuffiness of the elastic layers is equal $K_{0}=K_{1}=\ldots=K_{i}=\ldots=K_{m-1}=K_{m}=K$. Finally, we obtain the system of $m$ algebraic equations as:

$$
\begin{aligned}
S_{n} W_{1 n}-v_{n} W_{2 n}=0, & i=1, \\
-v_{n} W_{i-1 n}+S_{n} W_{i n}-v_{n} W_{i+1 n}=0, & i=2,3, . . m-1, \\
-v_{n} W_{m-1 n}+S_{n} W_{m n}=0, & i=m,
\end{aligned}
$$

or in the matrix form as: 


$$
\left[\begin{array}{ccccccccccc}
S_{n} & -v_{n} & 0 & \ldots & 0 & 0 & 0 & \ldots & 0 & 0 & 0 \\
-v_{n} & S_{n} & -v_{n} & \ldots & 0 & 0 & 0 & \ldots & 0 & 0 & 0 \\
\ldots & \ldots & \ldots & \ldots & \ldots & \ldots & \ldots & \ldots & \ldots & \ldots & \ldots \\
0 & 0 & 0 & \ldots & S_{n} & -v_{n} & 0 & \ldots & 0 & 0 & 0 \\
0 & 0 & 0 & \ldots & -v_{n} & S_{n} & -v_{n} & \ldots & 0 & 0 & 0 \\
0 & 0 & 0 & \ldots & 0 & -v_{n} & S_{n} & \ldots & 0 & 0 & 0 \\
\ldots & \ldots & \ldots & \ldots & \ldots & \ldots & \ldots & \ldots & \ldots & \ldots & \ldots \\
0 & 0 & 0 & \ldots & 0 & 0 & 0 & \ldots & 0 & S_{n} & -v_{n} \\
0 & 0 & 0 & \ldots & 0 & 0 & 0 & \ldots & 0 & -v_{n} & S_{n}
\end{array}\right]\left\{\begin{array}{c}
W_{1 n} \\
W_{2 n} \\
W_{3 n} \\
\ldots \\
W_{i-1 n} \\
W_{i n} \\
W_{i+1 n} \\
\ldots \\
W_{m-2 n} \\
W_{m-1 n} \\
W_{m n}
\end{array}\right\}=\left\{\begin{array}{c}
0 \\
0 \\
0 \\
\ldots \\
0 \\
0 \\
0 \\
\ldots \\
0 \\
0 \\
0
\end{array}\right\}
$$

where:

$$
\begin{gathered}
S_{n}=-\Omega_{n}^{2} \xi_{n}+2 v_{n}+\boldsymbol{\alpha}_{n}^{4}-\boldsymbol{\alpha}_{n}^{2}\left(\bar{F}-\bar{N}_{\boldsymbol{\theta}}\right) \xi_{n}, \\
v_{n}=K \boldsymbol{\xi}_{n}, \\
\xi_{n}=1+\eta^{2} \boldsymbol{\alpha}_{n}^{2},
\end{gathered}
$$

Analytical solution of the homogeneous system of algebraic equations (13) is available only for the case when MNBS is composed of identical nanobeams and coupling layers. Based on the methodology presented in the papers Karličić et al. [29, 30], solution of the $i$ th algebraic equation can be assumed in the following form:

$$
W_{i n}=N \cos \left(i \varphi_{c c}\right)+M \sin \left(i \varphi_{c c}\right), \quad i=1,2, \ldots m,
$$

Substituting Eq. (15) into the $i$-th algebraic equation of the system (13), we obtain two trigonometric equations, by assuming that constants $M$ and $N$ are not simultaneously equal to zero:

$$
\begin{array}{ll}
N\left\{-v_{n} \cos \left[(i-1) \varphi_{c c}\right]+S_{n} \cos \left(i \varphi_{c c}\right)-v_{n} \cos \left[(i+1) \varphi_{c c}\right]\right\}=0, & i=2, \ldots m-1, \\
M\left\{-v_{n} \sin \left[(i-1) \varphi_{c c}\right]+S_{n} \sin \left(i \varphi_{c c}\right)-v_{n} \sin \left[(i+1) \varphi_{c c}\right]\right\}=0, & i=2, \ldots m-1,
\end{array}
$$

wherefrom we obtain the following system of equations:

$$
\begin{array}{ll}
\left(S_{n}-2 v_{n} \cos \varphi_{c c}\right) N \cos \left(i \varphi_{c c}\right)=0, & i=2, \ldots m-1, \\
\left(S_{n}-2 v_{n} \cos \varphi_{c c}\right) M \sin \left(i \varphi_{c c}\right)=0, & i=2, \ldots m-1,
\end{array}
$$

From the above system of equations we can conclude that, $N \neq 0, \cos \left(i \varphi_{c c}\right) \neq 0$ or $M \neq 0, \sin \left(i \varphi_{c c}\right) \neq 0$ when the system has an oscillatory behavior for $i=2, \ldots m-1$. Finally, we can obtain the frequency equation:

$$
S_{n}=2 v_{n} \cos \varphi_{c c} .
$$


where $\boldsymbol{\varphi}_{\mathrm{cc}}$ is unknown parameter determined by replacing the solutions from Eq. (15) in the first and the last equation of the system of equations (13). Using the methodology presented in the papers proposed by Rašković [27], Stojanović et al. [28] and Karličić et al. [29 and 30], we obtain the solutions for unknown parameter $\boldsymbol{\varphi}_{c c, s}$ in "Clamped-Chain" systems as:

$$
\boldsymbol{\varphi}_{c c, s}=\frac{s \pi}{m+1}, \quad s=1,2,3, \ldots, m .
$$

Introducing the expression for $\boldsymbol{\varphi}_{c c, s}$ and Eqs. (14) into Eq. (18), and assuming axial compressive load $\bar{F}=0$, we get the natural frequencies in the dimensionless form as:

$$
\Omega_{n c c, s}=\sqrt{\frac{2 v_{n}\left(1-\cos \boldsymbol{\varphi}_{c c, s}\right)+\boldsymbol{\alpha}_{n}^{4}+\boldsymbol{\alpha}_{n}^{2} \bar{N}_{\theta} \xi_{n}}{\xi_{n}}}, \quad s=1,2, . ., m .
$$

The analytical form of the critical buckling load under the influence of temperature change can be obtained by introducing the expression for $\boldsymbol{\varphi}_{c c, s}$ and Eqs. (14) into Eq. (18) and assuming that the natural frequencies of system $\Omega_{n}$ are equal to zero. Then the critical buckling load is of the form:

$$
\bar{F}_{n c c, s}=\frac{2 v_{n}\left(1-\cos \boldsymbol{\varphi}_{c c, s}\right)+\boldsymbol{\alpha}_{n}^{4}}{\boldsymbol{\alpha}_{n}^{2} \xi_{n}}+\bar{N}_{\boldsymbol{\theta}}, \quad s=1,2, . ., m .
$$

Finally, in the case when the number of nanobeams in MNBS increases towards infinity i.e. taking $m \rightarrow \infty$ into Eqs. (22) and (23), we obtain asymptotic or critical natural frequencies and critical buckling loads in the following form:

$$
\begin{gathered}
\Omega_{\substack{n c c, s \\
m \rightarrow \infty}}=\sqrt{\frac{\boldsymbol{\alpha}_{n}^{4}+\boldsymbol{\alpha}_{n}^{2} \bar{N}_{\theta} \xi_{n}}{\xi_{n}},} \\
\bar{F}_{\substack{n c c, s \\
m \rightarrow \infty}}=\frac{\boldsymbol{\alpha}_{n}^{4}}{\boldsymbol{\alpha}_{n}^{2} \xi_{n}}+\bar{N}_{\theta},
\end{gathered}
$$

Now, we can conclude that the asymptotic natural frequencies and the critical buckling loads represented by expressions (22) and (23), respectively, are dependent only on the system of material parameters and temperature change.

\section{NUMERICAL RESULTS}

In order to examine the effects of material parameters, such as stiffness coefficient of elastic medium, temperature change and nonlocal parameter on natural frequencies and critical buckling loads of the nonlocal elastic MNBS, numerical examples are presented and discussed here. In addition, the influence of temperature change at low and high temperatures on the vibration and stability response of MNBS is studied. The physical and geometrical parameters used in numerical simulations are adopted from the paper by Murmu and Pradhan $[25,26]$. In the following parametric study, we observe changes of the lowest natural frequency and the critical buckling load of the system for a single vibration mode. 

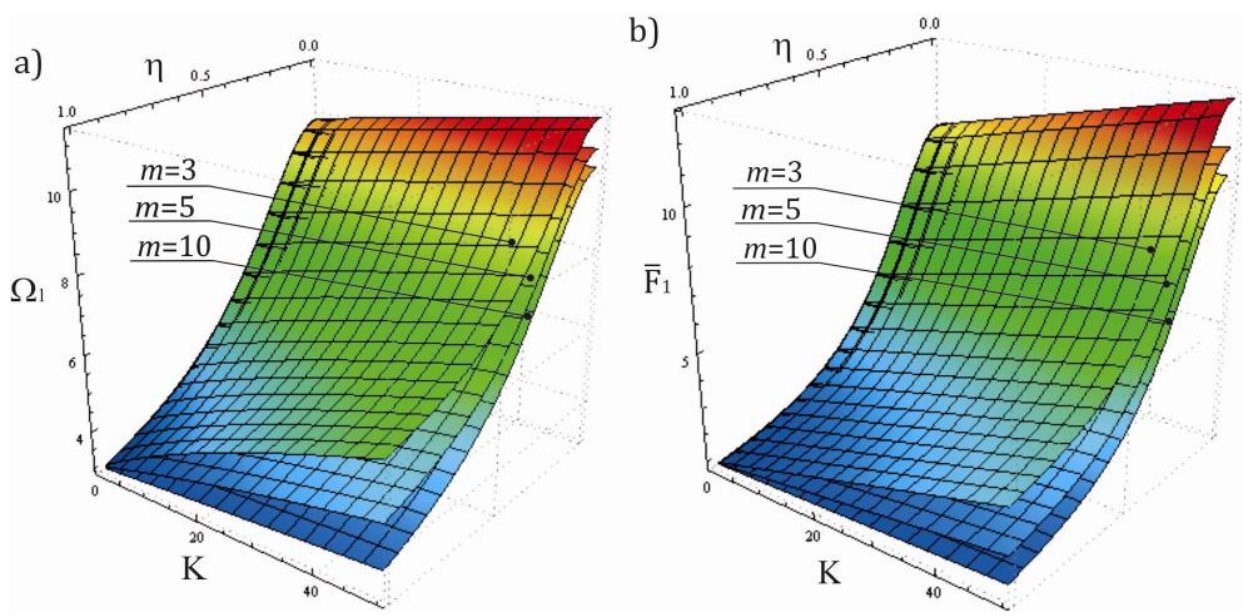

Fig. 2 The influence of the nonlocal parameter and stiffness of the Winkler elastic medium on a) natural frequencies and b) critical buckling load for different number of nanobeams

The first natural frequency and the critical buckling load of MNBS versus the nonlocal parameter and stiffness of the Winkler elastic medium, for different numbers of nanobeams, are shown in Fig. 2. As expected, an increase in the nonlocal parameter leads to a decrease of the natural frequency and the critical buckling load of the system. From the same figure, one can observe that the stiffness of the Winkler's elastic medium $K$ has a very strong "hardening" effect on the natural frequency and the critical buckling load. Further, one can see that an increase of the number of nanobeams $(m=3,5,10)$ in MNBS leads to a decrease of both the natural frequency and the critical buckling load towards certain critical values. This is in line with expressions (22) and (23) and the results obtained in papers by Karličić et al. [29, 30].

a)

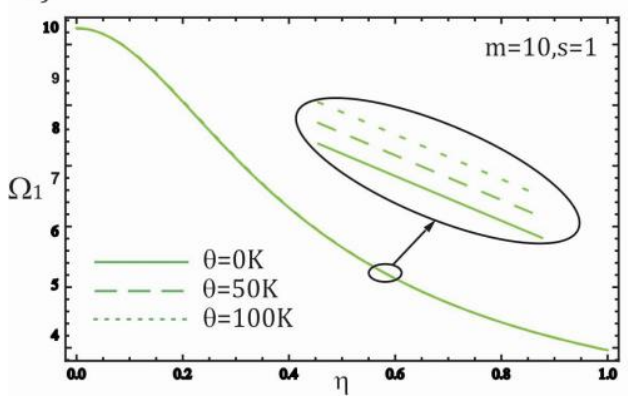

b)

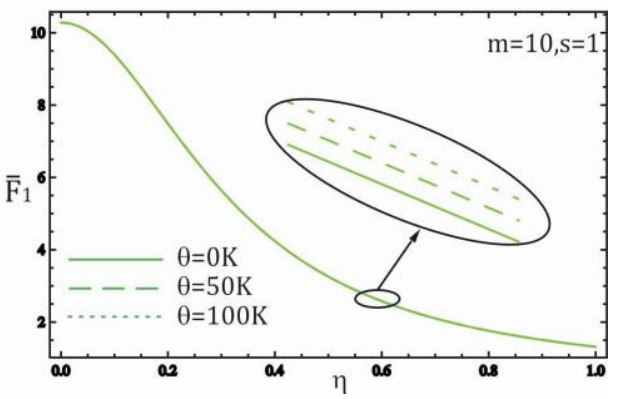

Fig. 3 The influence of the nonlocal parameter and temperature change at low or room temperatures on the first a) natural frequency and b) critical buckling load 
a)

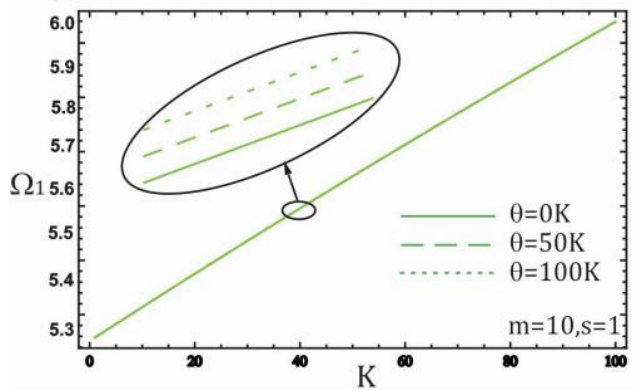

b)

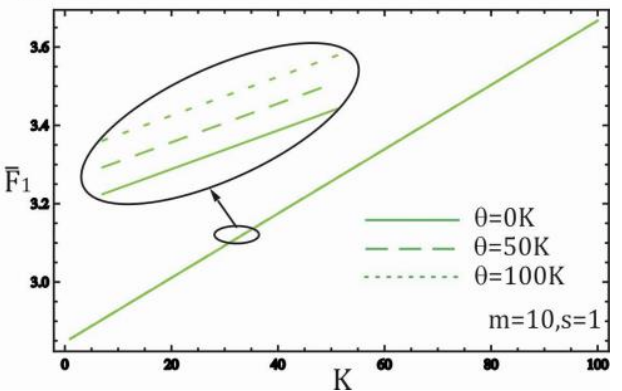

Fig. 4 The influence of the stiffness coefficients and temperature change at low or room temperatures on the first a) natural frequency and b) critical buckling load

a)

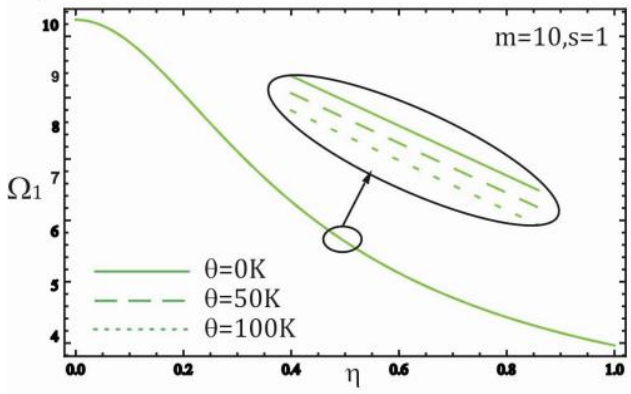

b)

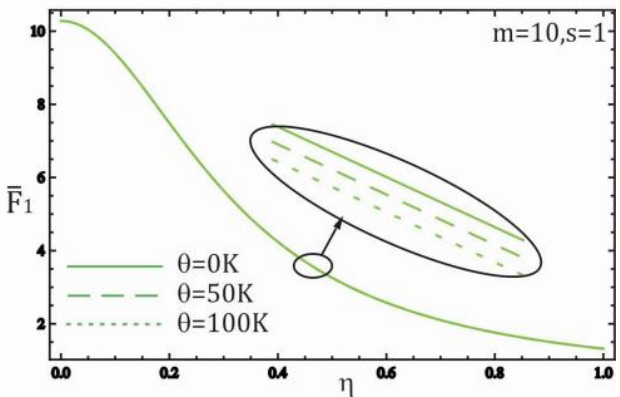

Fig. 5 The influence of the nonlocal parameter and temperature change at high temperatures on the first a) natural frequency and b) critical buckling load

a)

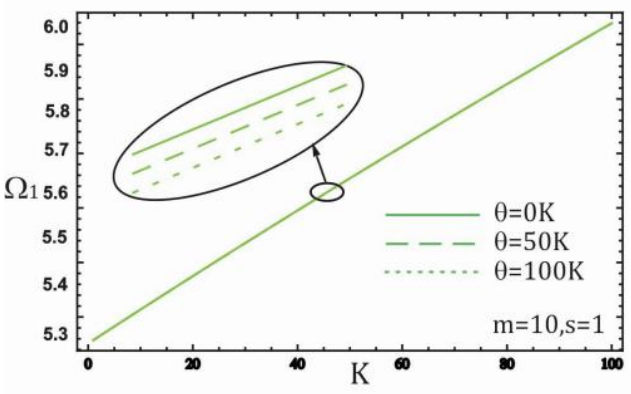

b)

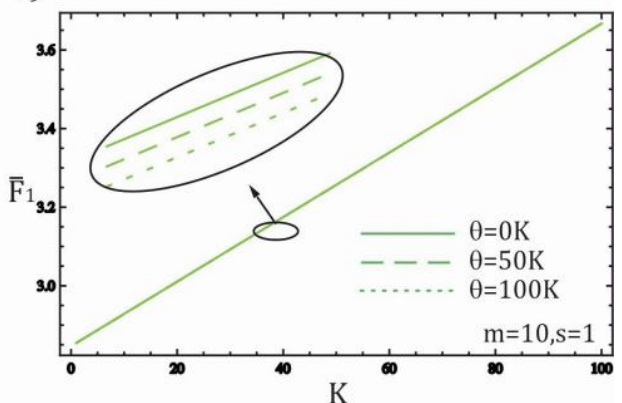

Fig. 6 The influence of the stiffness coefficients and temperature change at high temperatures on the first a) natural frequency and b) critical buckling load

Figs. 3-6 shows the influence of temperature change $\theta$ at low temperature $\left(\boldsymbol{\alpha}_{x}=-1.6 \times 10^{-6} K^{-1}\right)$ and high temperature $\left(\boldsymbol{\alpha}_{x}=1.1 \times 10^{-6} K^{-1}\right)$ environment (see Murmu and Pradhan $[25,26])$ on the natural frequency and the critical buckling load for different 
values of nonlocal parameter and stiffness coefficient of elastic medium. It should be noted that we consider the first natural frequency and the critical buckling load of MNBS composed of ten elastically connected nanobeams $(m=10)$ coupled in the "Clamped - Chain" system. To illustrate the effect of temperature change at low temperature environment with the natural frequency and the critical buckling load of MNBS, numerical experiments are carried out for different values of nonlocal parameter and stiffness coefficients and three different values of temperature change $\theta=0.50$ and $100 K$. From Fig. 3 it can be observed that an increase of temperature change leads to an increase in both the natural frequency and the critical buckling load. Fig. 4 illustrates the influence of a changing stiffness coefficient of elastic medium with the natural frequency and the critical buckling load of MNBS for three different values of temperature change. From this figure, it is also found that the natural frequency and the critical buckling load are nearly linearly dependent on stiffness coefficient $K$. In addition, it can be observed that an increase of stiffness coefficient increases the natural frequency and the critical buckling load. Moreover, the influence of temperature changes is small, but not negligible. The similar behavior can be observed in the previous case presented in Fig. 3. Finally, one can conclude that the temperature change at low temperature environment causes an increase of the system's stiffness, which leads to an increase of the natural frequencies and the buckling loads of the system. The obtained results are also in line with the results obtained in papers by Murmu and Pradhan $[25,26]$.

The effects of temperature change at higher temperature environment with the natural frequency and the critical buckling load of MNBS embedded in elastic medium where $m=10$ and $s=1$ are shown in Figs. 5 and 6. It can be noticed that temperature change has a different influence on the system's natural frequency and its critical buckling load than in the case of low temperature environment. Here, one can notice that an increase of temperature change leads to a decrease of both the natural frequency and the critical buckling load. From the physical viewpoint, this means that the total stiffness of MNBS decreases for an increase of temperature. Regarding the influence of nonlocal parameter and stiffness coefficient of elastic medium on the vibration and stability response of MNBS it is similar as in the case of low temperature environment.

Molecular dynamic simulation studies analyzing the dynamical and stability behavior of systems with a larger number of coupled nanobeams with thermal effects are not yet published in the literature. However, the authors have found MD simulation results for vibration and vibration of a single-walled carbon nanotube presented by Ansari et al. [34]. For MNBS it is well known that the lowest natural frequency and the buckling load of such system represent the fundamental frequency and critical buckling load and it is independent of the influence of a number of nanobeams in the system and chain coupling conditions, see Karličić et al. [33]. The fundamental frequency and the critical buckling load are equivalent to the natural frequency and the buckling load of a single nanobeam. Thus, our results for the lowest natural frequency and the critical buckling load of MNBS without thermal change can be used to validate them with the results obtained for free vibration of a single-walled carbon nanotube via molecular dynamics simulation in Ansari et al. [34]. This is also in line with expressions for asymptotic natural frequency given in Eq. (22). The mechanical properties of single-walled carbon nanotube considered in this comparative study are adopted from Ansari et al. [34]. Table 1 shows the fundamental natural frequencies obtained by using the molecular dynamic simulation and nonlocal continuum mechanics approach for different values of the aspect ratios L/D. From Table 
1, it can be noticed that the results obtained by using the trigonometric method are in excellent agreement with the results presented by Ansari et al. [34].

Table 1 Comparison fundamental frequencies (THz) presented analytical solution with results obtained in the Ansari et al. [34]

\begin{tabular}{ccccccc}
\hline \multicolumn{7}{c}{$\begin{array}{c}\text { Timoshenko beam theory } \\
\text { Ansari et al. [34] }\end{array}$} \\
\hline L/D & $\begin{array}{c}\text { MD } \\
\text { simulation }\end{array}$ & $\begin{array}{c}\text { Classical } \\
\text { theory }\end{array}$ & $\begin{array}{c}\text { Stress } \\
\text { gradient } \\
\text { theory }\end{array}$ & $\begin{array}{c}\text { Strain } \\
\text { gradient } \\
\text { theory }\end{array}$ & $\begin{array}{c}\text { Strain/ inertia } \\
\text { gradient } \\
\text { theory }\end{array}$ & $\begin{array}{c}\text { Nonlocal } \\
\text { theory }\end{array}$ \\
\hline 8.3 & 0.5299 & 0.5306 & 0.5302 & 0.5302 & 0.5299 & 0.5485 \\
10.1 & 0.3618 & 0.3606 & 0.3604 & 0.3604 & 0.3603 & 0.3707 \\
13.7 & 0.1931 & 0.1972 & 0.1971 & 0.1971 & 0.1971 & 0.2016 \\
17.3 & 0.1103 & 0.1240 & 0.1240 & 0.1240 & 0.1240 & 0.1264 \\
20.9 & 0.0724 & 0.0851 & 0.0851 & 0.0851 & 0.0851 & 0.0860 \\
24.5 & 0.0519 & 0.0620 & 0.0620 & 0.0620 & 0.0620 & 0.0630 \\
28.1 & 0.0425 & 0.0471 & 0.0471 & 0.0471 & 0.0471 & 0.0479 \\
31.6 & 0.0358 & 0.0373 & 0.0373 & 0.0373 & 0.0373 & 0.0379 \\
35.3 & 0.0287 & 0.0299 & 0.0299 & 0.0299 & 0.0299 & 0.0303 \\
39.1 & 0.0259 & 0.0244 & 0.0244 & 0.0244 & 0.0244 & 0.0247 \\
\hline
\end{tabular}

\section{CONCLUSIONS}

In the present paper, the vibration and the stability properties of MNBS embedded in Winkler elastic medium for different number of simply supported nanobeams are studied. The system of $m$ partial differential equations is derived using the D' Alembert principle and nonlocal thermo-elastic constitutive relation. The possibility of using nanostructures such as CNTs, $\mathrm{ZnO}$ or BN nanotubes as nanoresonators, nanosensors and in other NEMS devices demands exploration of natural frequencies of single or multiple nanostructure systems. However, external field effects, such as temperature, or the influence of surrounding medium, can significantly shift the values of natural frequencies. Thus, such effect needs to be taken into account to obtain, as much as possible, realistic models to simulate the mechanical behavior of nano-scale systems. Nonlocal continuum theory, which considers size-effects, is one of the most convenient theories to deal with a large nano-scale system with various external field effects, easily included into a model. The nonlocal EulerBernoulli beam theory takes into account the length-scale i.e. nonlocal parameter, where thermal effects are also introduced through constitutive relation. The Bernoulli - Fourier and trigonometric methods are used to obtain closed form analytical solutions for thermal vibration and stability response of the proposed system. The influence of temperature changes and nonlocal parameter with natural frequencies and critical buckling loads are considered. In addition, the influence of stiffness of the elastic medium $K$, on both of mechanical responses of MNBS, is investigated through numerical examples. It is found that the natural frequency and the critical buckling load of MNBS are strongly influenced by nonlocal and stiffness coefficients. Further, significant influence of the temperature change on the natural frequency and the critical buckling load can also be recognized. From the obtained results, one can conclude that the nonlocal parameter and the temperature change at 
high temperature environment are having dampening properties on the natural frequency and the critical buckling load. On the other hand, an increase of stiffness coefficients of the elastic medium and temperature change at low temperature environment leads to an increase of total stiffness of MNBS and consequently to an increase of the natural frequency and the critical buckling load.

Acknowledgements: This research was supported by the research grant of the Serbian Ministry of Science and Environmental Protection under the number of ON 174001 and ON 174011.

\section{REFERENCES}

1. Bezryadin, A., Verschueren, A. R. M., Tans, S. J., Dekker, C., 1998, Multiprobe transport experiments on individual single-wall carbon nanotubes, Physical Review Letters, 80(18), pp.4036- 4039.

2. Gogotsi, Y., Libera, J. A., Güvenç-Yazicioglu, A., Megaridis, C. M., 2001, In situ multiphase fluid experiments in hydrothermal carbon nanotubes, Applied physics letters, 79(7), pp. 1021-1023.

3. Lu, J. P., 1997, Elastic properties of carbon nanotubes and nanoropes, Physical Review Letters, 79 (7), pp. 1297-1306.

4. Werder, T., Walther, J. H., Jaffe, R. L., Halicioglu, T., Koumoutsakos, P., 2003, On the water-carbon Interaction for use in molecular dynamics simulations of graphite and carbon nanotubes, The Journal of Physical Chemistry B, 107(6), pp. 1345-1352.

5. Wen Xing, B., ChangChun, Z., WanZhao, C., 2004, Simulation of Young's modulus of single-walled carbon nanotubes by molecular dynamics, Physica B: Condensed Matter, 352(1), pp. 156-163.

6. Liew, K. M., He, X. Q., Wong, C. H., 2004, On the study of elastic and plastic properties of multi-walled carbon nanotubes under axial tension using molecular dynamics simulation, Acta Materialia, 52(9), pp. 2521-2527.

7. Neyts, E. C., Shibuta, Y., van Duin, A. C., Bogaerts, A., 2010, Catalyzed growth of carbon nanotube with definable chirality by hybrid molecular dynamics - force biased Monte Carlo simulations, ACS nano, 4(11), pp. 6665-6672.

8. Ansari, R., Gholami, R., Sahmani, S., 2013, Size-dependent vibration of functionally graded curved microbeams based on the modified strain gradient elasticity theory, Archive of Applied Mechanics, 83(10), pp. 1439-1449.

9. Chen, W. J., Li, X. P., 2013, Size-dependent free vibration analysis of composite laminated Timoshenko beam based on new modified couple stress theory, Archive of Applied Mechanics, 83(3), pp. 431-444

10. Challamel, N., Zhang, Z., Wang, C.M., Reddy, J.N., Wang, Q., Michelitsch, T., Bernard, C., 2014, On nonconservativeness of Eringen's nonlocal elasticity in beam mechanics: correction from a discrete-based approach, Archive of Applied Mechanics, DOI 10.1007/s00419-014-0862-x.

11. Natsuki, T., Endo, M., 2004, Stress simulation of carbon nanotubes in tension and compression, Carbon, 42(11), pp. 2147-2151.

12. Gao, Y., Wang, Z. L., 2007, Electrostatic potential in a bent piezoelectric nanowire. The fundamental theory of nanogenerator and nanopiezotronics, Nano letters, 7(8), pp. 2499-2505.

13. Chopra, N. G., Luyken, R. J., Cherrey, K., Crespi, V. H., Cohen, M. L., Louie, S. G., Zettl, A., 1995, Boron nitride nanotubes, Science, 269(5226), pp. 966-967.

14. Loiseau, A., Willaime, F., Demoncy, N., Schramchenko, N., Hug, G., Colliex, C., Pascard, H., 1998, Boron nitride nanotubes, Carbon, 36(5), pp. 743-752.

15. Eringen, A. C., 1972, Nonlocal polar elastic continua, International Journal of Engineering Science, 10(1), pp. $1-16$.

16. Eringen, A. C., Edelen, D. G. B., 1972, On nonlocal elasticity, International Journal of Engineering Science, 10(3), pp. 233-248.

17. Eringen, A. C., 1992, Vistas of nonlocal continuum physics, International Journal of Engineering Science, 30(10), pp. 1551-1565.

18. Eringen, A. C., 1983, On differential equations of nonlocal elasticity and solutions of screw dislocation and surface waves, Journal of Applied Physics, 54(9), pp. 4703-4710. 
19. Peddieson, J., Buchanan, G. R., McNitt, R. P., 2003, Application of nonlocal continuum models to nanotechnology, International Journal of Engineering Science, 41(3), pp. 305-312.

20. Reddy, J. N., Pang, S. D., 2008, Nonlocal continuum theories of beams for the analysis of carbon nanotubes, Journal of Applied Physics, 103(2), pp. 023511-16.

21. Reddy, J. N., 2007, Nonlocal theories for bending, buckling and vibration of beams, International Journal of Engineering Science, 45(2), pp. 288-307.

22. Zhang, Y. Q., Liu, X., Liu, G. R., 2007, Thermal effect on transverse vibrations of double-walled carbon nanotubes, Nanotechnology, 18(44), 445701 (7pp).

23. Zhang, Y. Q., Liu, X., Zhao, J. H., 2008, Influence of temperature change on column buckling of multiwalled carbon nanotubes, Physics Letters A, 372(10), pp. 1676-1681.

24. Janghorban, M., 2012, Two different types of differential quadrature methods for static analysis of microbeams based on nonlocal thermal elasticity theory in thermal environment, Archive of Applied Mechanics, 82(5), pp. 669-675.

25. Murmu, T., Pradhan, S. C., 2009, Thermo-mechanical vibration of a single-walled carbon nanotube embedded in an elastic medium based on nonlocal elasticity theory, Computational Materials Science, 46(4), pp. 854-859.

26. Murmu, T., Pradhan, S. C., 2010, Thermal effects on the stability of embedded carbon nanotubes, Computational Materials Science, 47(3), pp. 721-726.

27. Rašković, D., 1963, Small forced damping vibrations of homogeneous torsional system with special static constrints. Publications de l'Institut Mathématique, 3(17), pp. 27-34.

28. Stojanović, V., Kozić, P., Janevski, G., 2013, Exact closed-form solutions for the natural frequencies and stability of elastically connected multiple beam system using Timoshenko and high-order shear deformation theory. Journal of Sound Vibration, 332(3), pp. 563-576.

29. Karličić, D., Kozić, P., Pavlović, R., 2014, Free transverse vibration of nonlocal viscoelastic orthotropic multinanoplate system (MNPS) embedded in a viscoelastic medium, Composite Structures, 115, pp. 89-99.

30. Karličić, D., Adhikari, S., Murmu, T., Cajić, M., 2014, Exact closed-form solution for non-local vibration and biaxial buckling of bonded multi-nanoplate system, Composites Part B: Engineering, 66, pp. 328-339.

31. Ansari, R., Rouhi, H., Sahmani, S., 2011, Calibration of the analytical nonlocal shell model for vibrations of double-walled carbon nanotubes with arbitrary boundary conditions using molecular dynamics, International Journal of Mechanical Sciences, 53(9), pp. 786-792.

32. Frank, I. W., Deotare, P. B., McCutcheon, M. W., Lončar, M., 2010, Programmable photonic crystal nanobeam cavities, Optics Express, 18(8), pp. 8705-8712.

33. Karličić, D., Murmu, T., Cajić, M., Kozić, P., Adhikari, S., 2014, Dynamics of multiple viscoelastic carbon nanotube based nanocomposites with axial magnetic field, Journal of Applied Physics, 115(23), pp. 234303-14

34. Ansari, R., Gholami, R., Rouhi, H., 2012, Vibration analysis of single-walled carbon nanotubes using different gradient elasticity theories, Composites Part B: Engineering, 43(8), pp. 2985-2989. 